\section{Modified Anderson-Darling detector for Spectrum Sensing}

\section{Bart Scheers, Djamel Teguig and Vincent Le Nir}

Goodness-of-Fit (GoF) testing is a promising approach for blind spectrum sensing in cognitive radio (CR) applications. In this paper, we present a modification on the Anderson-Darling detector by taking into account the physical characteristics of spectrum sensing, resulting in a reduction of the false alarm rate by a factor of two for a given detection threshold in the binary hypothesis test.

Introduction: A GoF test is a blind non parametric hypothesis test which can be used to detect the presence of signals in noise by determining whether the received samples are (are not) drawn from a distribution with a Cumulative Distribution Function $(\mathrm{CDF}) F_{0}$, representing the noise CDF $[1,2,3]$. The hypothesis to test whether or not a signal is present can be formulated as follows:

$$
\left\{\begin{array}{l}
H_{0}: F_{n}(x)=F_{0}(x) \\
H_{1}: F_{n}(x) \neq F_{0}(x),
\end{array}\right.
$$

where $F_{0}$ represents the hypothesized noise CDF. $F_{n}(x)$ is the empirical $\mathrm{CDF}$ of the received sample and can be calculated by:

$$
F_{n}(x)=\left|\left\{i: x_{(i)} \leq x, 1 \leq i \leq n\right\} / n\right|,
$$

where |.| indicates cardinality, $x_{(i)}$ are the ordered samples under test $\left(x_{1} \leq x_{2} \leq \ldots \leq x_{n}\right)$ and $n$ represents the total number of samples.

In statistics, the aim of $\mathrm{GoF}$ is to test the hypothesis that samples have been drawn from a population with a specified continuous CDF $F_{0}(x)$. This means that in the $H_{1}$ hypothesis, for a given value of $x$, the discrepancy between $F_{n}(x)$ and the hypothetical $F_{0}(x)$ can either be positive or negative (the empirical CDF $F_{n}(x)$ can be left or right from $F_{0}(x)$ in a graphical representation). However, in the application of spectrum sensing this is not necessarily true, as will be shown in this paper.

The remainder of this paper is organized as follows. In the next section, the Anderson-Darling (AD) test statistic is detailed, along with a description of how the AD detector can be used for spectrum sensing. In the following section, the shortcoming of the $\mathrm{AD}$ test for spectrum sensing is highlighted. In the fourth section, a modification on the AD detector is proposed, taking into account the physical characteristics of spectrum sensing. Finally the performance of the modified detector will be evaluated by simulations.

The Anderson-Darling test: The commonly used test statistic to perform GoF testing was proposed by T. W. Anderson and D. A. Darling in 1952 [5].

$$
\frac{1}{n} A_{n}^{2}=\int_{0}^{1}\left[F_{n}(x)-F_{0}(x)\right]^{2} \psi\left[F_{0}(x)\right] d F_{0}(x),
$$

This test statistic is based on the Von Mises criterion, which is an average of the squared discrepancy $\left[F_{n}(x)-F_{0}(x)\right]^{2}$, weighted by the increase in $F_{0}(x)$. The authors in [5] proposed an extra weighting function $\psi\left(F_{0}(x)\right)$ to give more importance to the tails of the CDF.

$$
\psi\left[F_{0}(x)\right]=\frac{1}{F_{0}(x)\left[1-F_{0}(x)\right]}
$$

The test statistic given in (3) can be numerically calculate as [6]

$$
A_{n}^{2}=-n-\frac{\sum_{i=1}^{n}(2 i-1)\left(\ln F_{0}\left(x_{(i)}\right)+\ln \left(1-F_{0}\left(x_{(n+1-i)}\right)\right)\right)}{n} .
$$

In Software Defined Radio technology, the received baseband samples in the digital domain are complex in nature. In this case, the most practical approach to apply the $\mathrm{AD}$ test for spectrum sensing is to consider the squared magnitude of the complex samples (i.e. energy of the samples) and test their empirical distribution against the hypothetical noise energy distribution [3]. The sensing method can be summarized as follows: Consider the classical binary hypothesis test

$$
\left\{\begin{array}{l}
H_{0}: x_{i}=w_{i} \\
H_{1}: x_{i}=r_{i}+w_{i}
\end{array}\right.
$$

where $r_{i}$ are the received complex samples of the transmitted signal, filtered by the channel, and $w_{i}$ is the complex noise. We now consider the non-negative random variable $Y_{i}=\left|x_{i}\right|^{2}$ which corresponds to the received energy and construct the empirical CDF $F_{n}$ as given in (2). We assume that $F_{0}$, representing the hypothetical noise energy distribution, is known or can be estimated. Next, the test statistic $A_{n}^{2}$ is calculated by (5). Once $A_{n}^{2}$ is computed, it will be compared to a predefined threshold $\lambda$ :

$$
\left\{\begin{array}{l}
H_{0}: A_{n}^{2} \leq \lambda \\
H_{1}: A_{n}^{2}>\lambda
\end{array}\right.
$$

The value of $\lambda$ is determined for a specific value of $P_{f a}$. A table listing values of $\lambda$ corresponding to different false alarm probabilities $P_{f a}$ is given in [4]. Otherwise, these values can be computed in advance by Monte Carlo approach. Note that the distribution of $A_{n}^{2}$, and hence the value of $\lambda$ is independent of the noise distribution represented by $F_{0}$.

Problem statement: The problem with the AD test (and also with the Von Mises test) is that the deviation of the empirical CDF $F_{n}(x)$ to the reference $\mathrm{CDF} F_{0}(x)$ can be either to the left or to the right as the tests are based on the square of the difference $\left.\left[F_{n}(x)-F_{0}(x)\right)\right]^{2}$. For most statistical tests, this is convenient, but it is not the case for the application of spectrum sensing.

To illustrate this, let us consider the following simple example. Suppose we want to detect a signal in Gaussian noise. In this case, as the test is performed on the squared values of the received signal, $F_{0}$ will be the CDF of the $\chi_{2}^{2}$ distribution.

In figure 1 the result of 1000 Monte-Carlo simulations is shown under the $H_{0}$ hypothesis (no signal present). For each simulation the binary test (7) is performed with $\lambda=3.89$, corresponding to a false alarm rate $P_{f a}=0.01$. The empirical CDFs that are found in the $H_{0}$ hypothesis are represented in blue. The reference $\mathrm{CDF} F_{0}$ for the GoF test corresponding to a $\chi_{2}^{2} \mathrm{CDF}$ is represented in green. The empirical CDFs that are found in the $H_{1}$ hypothesis are in red and represent false alarms. As one can see, in total there are nine false alarms, which corresponds approximately to the pre-setted $P_{f a}$. Out of the nine, five false alarms are situated to the left of the reference CDF $F_{0}$ and four to the right.

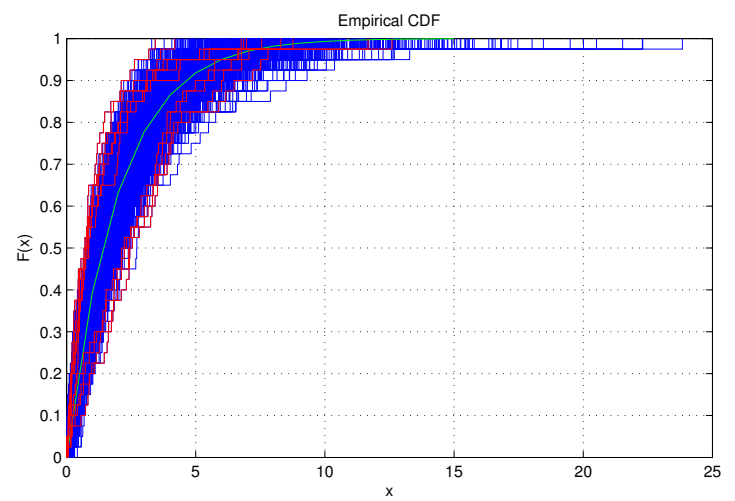

Fig. 1 Empirical CDFs of Gaussian noise realisations: in blue the empirical $C D F$ s found to be in the $H_{0}$ hypothesis, in red the empirical CDFs in the $H_{1}$ hypothesis. The reference $C D F F_{0}$ is represented in green.

In case of the proposed $\mathrm{AD}$ detector, $Y_{i}=\left|x_{i}\right|^{2}$ is a non-negative random variable. From statistics, it is known that the expected value of a non-negative random variable can be written as $E[Y]=\int_{0}^{\infty}(1-$ $\left.F_{Y}(x)\right) d x$. As the received signal $\left\{x_{i}\right\}$ has zero means, $E[Y]=$ $E\left[\left|x_{i}\right|^{2}\right]=\sigma_{x}^{2}$ also represents the received signal power. Hence, we find

$$
\sigma_{x}^{2}=\int_{0}^{\infty}\left(1-F_{Y}(x)\right) d x
$$


In other words, the received signal power equals the area of the region lying above the $\operatorname{CDF} F_{Y}(x)$ and below the line at height 1 to the right of the origin. Based on the same reasoning, we can state that the noise power $\sigma_{w}^{2}$ equals the area above $F_{0}$. This can be seen by replacing in (8) the signal $x_{i}$ by $w_{i}$ and $F_{Y}$ by the expected noise CDF $F_{0}$

Coming back to figure 1 , five empirical CDFs to the left of $F_{0}$ are found to be in the $H_{1}$ hypothesis by the $\mathrm{AD}$ detector. However, the area above these five empirical CDFs is inferior to the area above $F_{0}$. This means that for these empirical CDFs, the power in the received samples $x_{i}$ is less than the noise power. In the $H_{1}$ hypothesis, according to the Bienayme's formula, the power of the received signal $\sigma_{x}^{2}=\sigma_{r}^{2}+\sigma_{w}^{2}$, is always larger than the noise power, as signal and noise are uncorrelated. In other words, the five empirical CDFs to the left of $F_{0}$ that are found to be in the $H_{1}$ hypothesis can be excluded as false alarms. Tagging an empirical CDF left of $F_{o}$ as a possible signal has no physical meaning as it indicates that, according to (8), the power in the received samples would be less than the expected noise power. As signal and noise are uncorrelated, this is impossible.

Enhancement on the AD test statistic for Spectrum sensing: In order to take into account the observation stated in previous section, we modify the binary test given in (7) by adding a second condition to the $H_{1}$ hypothesis

$$
\left\{\begin{array}{l}
H_{1}: A_{n}^{2}>\lambda \text { and } \sigma_{x}^{2}>\sigma_{w}^{2}, \\
H_{0}: \text { otherwise }
\end{array}\right.
$$

In [5] it is shown that under the $H_{0}$ hypothesis, the random variable $\left[F_{n}(x)-F_{o}(x)\right]$ approaches a k-variate normal distribution as $n \rightarrow \infty$ with $E\left(F_{n}(x)-F_{o}(x)\right)=0$. This means that in the $H_{0}$ hypothesis, for a given value of $x$, statistically half of the empirical CDFs $F_{n}(x)$ will pass above $F_{o}(x)$ and half will pass underneath. Hence, statistically half of the false alarms are generated by empirical CDFs representing samples $x_{i}$ with a signal power $\sigma_{x}^{2}$ less than the expected noise power (i.e. CDFs mainly left of or above $F_{0}$ ) and half by empirical CDFs for which $\sigma_{x}^{2}$ is larger than the expected noise power (i.e. CDFs mainly to the right of $F_{0}$ ). By excluding in the modified binary test (9) the empirical CDFs which are to the left of $F_{0}$, i.e. empirical CDFs for which $\sigma_{x}^{2}>\sigma_{w}^{2}$ from the $H_{1}$ hypothesis, the false alarm rate will be reduced by a factor of 2 .

Simulation: To visualise this gain by a factor of 2 in $P_{f a}$, we compare in figure 2 the detection performance of both the classical and the modified $\mathrm{AD}$ detector as a function of $S N R$ in the range $-20 d B$ to $5 d B$. For the simulation, $\lambda$ is set to 2.495 for both detectors. The number of received samples $n$ to calculate the test statistics equals 40 . For very small values of SNR, the probability of detection $P_{d}$ will yield the value of $P_{f a}$. For the $\mathrm{AD}$ detector, according to [4], this value of $\lambda$ corresponds to a $P_{f a}=0.05$, which can be verified on figure 2. For the modified AD detector $P_{d}$ tends to 0.025 for low $S N R$ values, which is half of the presetted value of $P_{f a}$. We also observe that for higher values of $S N R$, the detection probability of both detectors are equal.

In figure 3 the receiver operating characteristic (ROC) curves of both detectors are represented for an $S N R$ value of $-3 d B$ and $n=40$. Also in these ROC-curves, the gain by the factor of 2 in $P_{f a}$ is very clear. For a given value of $P_{d}$, the modified $\mathrm{AD}$ detector, based on binary test (9) yields a $P_{f a}$ which is only half the $P_{f a}$ of the classic AD detector based on binary test (7). Vice-versa, for a given value of $P_{f a}$, the detection probability of the modified $\mathrm{AD}$ detector outperforms the detection probability of the AD detector. In summary we can state that for a given value of $P_{f a}$, the proposed detector is more sensitive than the classical AD detector.

Conclusion: In this paper we studied the Anderson-Darling test as a method for blind spectrum sensing. The $\mathrm{AD}$ detector will test the Goodness-of-Fit of empirical distribution of the squared magnitude of the received samples against the noise energy distribution, based on the $\mathrm{AD}$ test statistic $A_{n}^{2}$. In this test, the discrepancy between the empirical CDF and the reference $C D F$ can be either to the left or to the right. This means that in the $H_{0}$ hypothesis, empirical CDFs that are situated too much left of the reference CDF will also generate false alarms. This has no physical meaning in the application of spectrum sensing as this situation would corresponds to a signal with less power than the expected noise power. In the $H_{1}$ hypothesis, the overall power in the received signal should always be larger than the noise power, as noise and signal are uncorrelated. We therefore proposed a modified binary test to eliminate

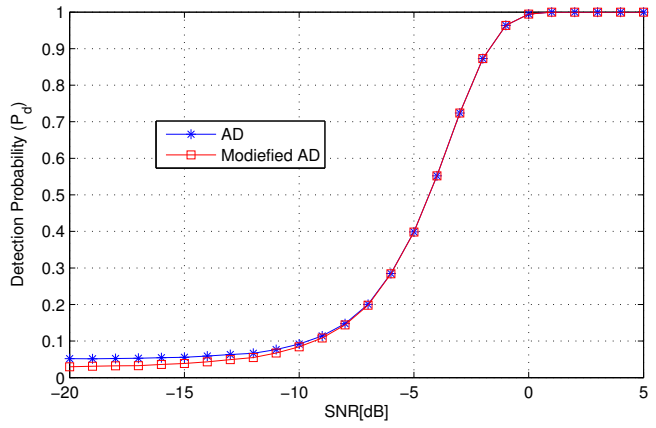

Fig. 2 Detection probability versus $S N R$ for $A D$ and Enhanced $A D$ detector $\lambda=2.495$ for both detectors, $n=40$

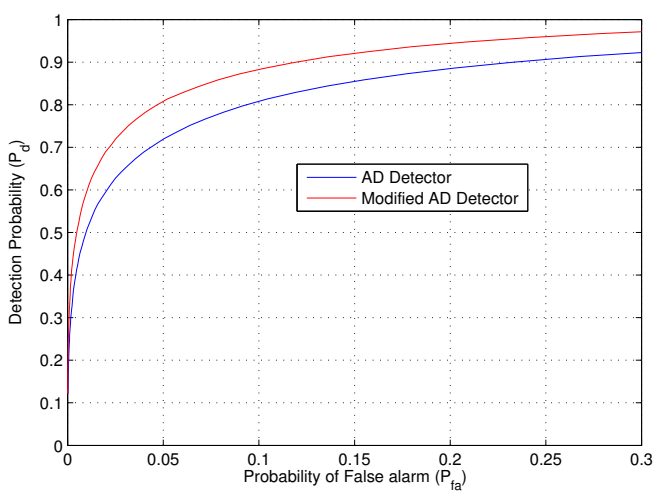

Fig. 3 Detection probability versus probability of false alarms for $A D$ and Enhanced $A D$ detector, $S N R=-3 d B, n=40$.

the empirical CDFs left to the reference $\mathrm{CDF}$ from the $H_{1}$ hypothesis, as we know that these empirical CDFs would correspond to a false alarm. Simulations have indeed shown that the modified AD detector reduces the false alarm rate by a factor 2 for a given threshold $\lambda$ or for a given probability of detection. As a consequence, for a given false alarm rate, the enhanced $\mathrm{AD}$ detector is more sensitive than the classic $\mathrm{AD}$ detector.

B. Scheers, D. Teguig and V. Le Nir (Royal Military Academy, Brussels, Belgium)

E-mail: bart.scheers@rma.ac.be

\section{References}

1 T. Kieu-Xuan, I. Koo: 'Cramer-von Mises test spectrum sensing for cognitive radio systems', Wireless Telecommunication Symposium, 2011.

2 H. Wang, E. H. Yang, Z. Zhao and W. Zhang: 'Spectrum sensing in cognitive radio using goodness of fit testing', IEEE Trans. Wireless Commun., vol. 8, no. 11, pp. 5427-5430, Nov. 2009.

3 D. Teguig, V. Le Nir and B. Scheers : 'Spectrum sensing method based on goodness of fit test using chi-square distribution', Electronics Letters, Volume 50, Issue 9, 24 April 2014, p. 713-715.

4 M. A. Stephens: 'EDF Statistics for Goodness of Fit and Some Comparisons', Journal of the American Statistic Association, vol.69, No.347, pp.730-737, Sep. 1974.

5 T. W. Anderson, D. A. Darling: 'Asymptotic Theory of Certain "Goodness of Fit" Criteria Based on Stochastic Processes', Annals of Mathematical Statistics, Vol. 23, pp.193-212,1952.

6 T. W. Anderson, D. A. Darling: 'A Test of Goodness of Fit', Journal of the American Statistical Association, Vol. 49, No. 268. , pp. 765-769,Dec. 1954. 